\title{
Predictors for 2-Year Functional and Morphological Outcomes of a Treat-and-Extend Regimen with Ranibizumab in Patients with Diabetic Macular Edema
}

\author{
Helena Giannakaki-Zimmermann ${ }^{a}$ Alexandra Behrndt ${ }^{a}$ b Laura Hoffmann $^{a}$ \\ Maria-Magdalena Guichard ${ }^{a}$ Cengiz Türksever ${ }^{a} \quad$ Christian Prünte $^{b, c, d}$ \\ Katja Hatz ${ }^{a, b}$ \\ aVista Klinik, Binningen, Switzerland; 'bFaculty of Medicine, University of Basel, Basel, Switzerland; 'Department of \\ Ophthalmology, University Hospital Basel, Basel, Switzerland; Institute of Molecular and Clinical Ophthalmology \\ Basel (IOB), Basel, Switzerland
}

\author{
Keywords \\ Spectral-domain optical coherence tomography - Diabetic \\ macular edema - Treat and extend - Outcome predictor . \\ Ranibizumab
}

\begin{abstract}
Purpose: The purpose of the study was to investigate longer term functional and morphological outcomes and their predictors in diabetic macular edema (DME) following a treatand-extend regimen (TER) without loading dose under ranibizumab. Methods: Patient data were reviewed and analyzed retrospectively over a period of 24 months after initiation of TER. Best-corrected visual acuity (BCVA), treatment frequency, and quantitative and qualitative spectraldomain optical coherence tomography parameters were assessed. Results: 118 eyes of 87 patients were included. A mean of $9.74 \pm 2.13$ injections in the first and $7.63 \pm 2.29$ in the second year were applied. There were significant gains of BCVA and reductions in central retinal thickness from baseline to 12 and 24 months (all $p<0.001$ ). Percentage of eyes with an intact inner segment/outer segment (IS/OS)
\end{abstract}

\begin{tabular}{ll}
\hline karger@karger.com & (c) 2021 The Author(s). \\
www.karger.com/ore & Published by S. Karger AG, Basel \\
& $\begin{array}{l}\text { This is an Open Access article licensed under the Creative Commons } \\
\text { Attribution-NonCommercial-4.0 International License (CC BY-NC) } \\
\text { (http://www.karger.com/Services/OpenAccessLicense), applicable to } \\
\text { the online version of the article only. Usage and distribution for com- } \\
\text { mercial purposes requires written permission. }\end{array}$
\end{tabular}

junction increased from $15.3 \%$ at baseline to $42.1 \%$ at 24 months $(p<0.001)$. An intact IS/OS junction at baseline increased the probability of having a dry retina after 12 months by $79.3 \%(p=0.017)$ and after 24 months by $88.1 \%(p=$ 0.040). Less IS/OS disruption at baseline predicted longer maximum recurrence-free treatment intervals at 2 years $(r=$ $-0.345, p<0.001)$ and better BCVA at 1 year $(r=-0.347, p<$ $0.001)$. Baseline bigger intraretinal cysts were associated with more IS/OS disruption at 24 months $(r=0.305, p=$ 0.007 ). Younger age and lower BCVA at baseline were predictive for a higher BCVA gain at 24 months $(p=0.046, p<$ 0.001). Conclusion: Ranibizumab applied in a TER without loading dose in DME significantly improves visual acuity and retinal anatomical structure throughout 2 years. The evaluated predictors might help guide routine clinical treatment in DME.

(C) 2021 The Author(s)

Published by S. Karger AG, Basel

Helena Giannakaki-Zimmermann and Alexandra Behrndt have contributed equally.
Katja Hatz

Head of Medical Retina and Research Department, Vista Klinik Binningen Hauptstrasse 55

$\mathrm{CH}-4102$ Binningen (Switzerland)

katja.hatz@vista.ch 


\section{Introduction}

Diabetic macular edema (DME) is the most common reason of permanent vision loss in patients with diabetes, and different treatment approaches have been discussed so far [1-4]. For a long time, retinal laser treatment was the standard treatment of DME, while later, the possibility of injecting agents, such as corticosteroids, intravitreally was discovered. The effectiveness of steroids was shown [5], but unfortunately, intravitreal steroid treatment can be associated with extensive side effects, such as the development of cataract or a rise in intraocular pressure $[6,7]$. Intravitreal implants such as Ozurdex ${ }^{\circledR}$ (dexamethasone) or Iluvien ${ }^{\circledR}$ (fluocinolone acetonide) have the same side effects and therefore are only used as a second-line treatment $[8,9]$. Currently, intravitreal injection of drugs targeting vascular endothelial growth factor (VEGF) is the gold standard for the treatment of DME [4, $10]$.

In the ranibizumab approval studies (RISE and RIDE) [11], patients received monthly injections during the whole study period. It was found that each patient reacted differently to the treatment. The authors postulated that may be not all patients might need a monthly treatment over the long term [11]. With the intent to individualize treatment, the Pro Re Nata (PRN) regimen was introduced: after an initial loading dose of 3-6 monthly injections, patients are examined monthly, and further treatment is applied based on retreatment criteria, such as new or ongoing activity of DME as evaluated by optical coherence tomography (OCT) and/or visual acuity criteria. The effectiveness of the PRN regimen was proven in the RESTORE study and in the DRCR Retina Network studies $[12,13]$. Even if with a PRN regimen, there are less injections; there are still monthly follow-up examinations. The treat-and-extend regimen (TER) is a possibility to simplify and individualize treatment even further. The TER was first introduced by Spaide for the treatment of neovascular age-related macular degeneration (AMD) [14]. The goal was to reduce the number of clinical visits and injections for each patient to a minimum, by identifying the longest possible individual recurrence-free treatment interval (RFTI). Several studies showed that the TER provided better visual acuity and less clinical visits in exudative AMD than PRN [15-17]. The TER principle was transferred to diseases other than AMD, such as macular edema due to retinal vein occlusion [18] and DME. Knowing that it is possible to keep DME under control for many patients with longer treatment intervals [19], the RETAIN study showed that the TER is not infe- rior to a PRN scheme in DME [20]. In the TREX-DME study, significantly fewer injections were applied in the TER groups than monthly treatment with similar visual and anatomic outcomes in all groups [21]. However, both prospective studies applied versions of the TER other than that usually used in clinical practice. Unfortunately, data from TER real-life settings in DME are limited so far, mainly due to low patient numbers in previously published studies [22-25].

Amendments in OCT technologies offer new details of retinal structures with very precise imaging also in DME [1]. Central retinal thickness (CRT) was first used to guide (re)treatments in both clinical trials and routine. But visual acuity seems not only to be influenced by retinal thickness [26]. Improved imaging quality, the followup mode, and eye tracking systems are able to detect small structural changes at the same OCT cross section of different visits and to find predictors for the course of TER patients.

To our knowledge, there is very little information on the efficacy of the TER in clinical routine with ranibizumab in DME behind year 1 and outcome-predicting factors. The aim of this study was to analyze longer term functional and morphological outcomes as well as outcomepredicting parameters in TER-treated patients with DME.

\section{Methods}

Study Design and Patients

This was a retrospective study at an ophthalmological tertiary referral center (VISTA Klinik - Ophthalmologic Clinic in Binningen, Basel-Land, Switzerland). Patients aged $>18$ years with either type 1 or 2 diabetes mellitus (as defined per the American Diabetes Association or World Health Organization [WHO] guidelines) and a diagnosed exudative maculopathy due to diabetic retinopathy treated with ranibizumab intravitreally following a TER without loading dose of at least 12 months in a setting of clinical routine were included in this study. Approval to conduct this study was obtained from the Local Ethics Committee (Ethikkommission Nordwestschweiz [EKNZ No. 2014-322]), and the study was performed in accordance with ICH-GCP guidelines and followed the tenets of the Declaration of Helsinki. The need for written consent from each individual patient was waived because of the retrospective nature of the study.

\section{Study Assessments and Treatments}

A chart review of consecutive patients fulfilling the aforementioned criteria and an initiation of treatment with ranibizumab using a TER between 2012 and 2015 were performed. Demographic data, pretreatment information, best-corrected visual acuity (BCVA), treatment intervals, and adverse events were collected for at least 1 year but up to 2 years from the date of their first TER treatment with ranibizumab. Snellen BCVA values were transformed into ETDRS scores according to Gregori et al. [27]. 
Within the clinical routine setting, OCT imaging was performed by experienced reading center-certified photographers using Spectralis SD-OCT (Heidelberg Engineering, Heidelberg, Germany; Software version 5.3). Scans were acquired using an established protocol consisting of volume scans. For the volume scan of $20 \times 15^{\circ}, 19$ frames spaced $247 \mu \mathrm{m}$ (high speed mode, 9 frames, and 512 A-scans) were acquired in a follow-up setting. Further, a 6-mm star scan (high speed mode, 9 frames, and 512 A-scans) was performed. Quantitative and qualitative evaluations were performed by 2 physicians (A.B. and L.H.) according to a standardized protocol.

For thickness analyses, a manual correction of the automated segmentations was performed if any segmentation errors were present. Inner segment/outer segment (IS/OS) and external limiting membrane (ELM) disruption score, as measured by SD-OCT, were evaluated, as described previously [28], by calculating the mean of the scores in the horizontal and vertical scans of the macular star setting. Here, the following grading was used: 0 , no disruption in 1 -mm center; 1 , mild disruption $<1 / 4$ within 1 -mm center; $2,1 / 4-3 / 4$ disruption within 1-mm center; and (3) $>3 / 4$ disruption within 1-mm center (see Figure 1a). By overlaying an ETDRS grid centered on the fovea, the central $1 \mathrm{~mm}$ was evaluated for disorganization of the retinal inner layers (DRIL) as defined by the inability to distinguish the boundaries between the ganglion cell/ inner plexiform layer complex, the inner nuclear layer, and the outer plexiform layer within $>50 \%$ of the central foveal $1 \mathrm{~mm}$. Hyperreflective foci (HRFs) were calculated as the sum of the HRF counts within the horizontal and vertical scans within the central $1 \mathrm{~mm}$. The height of the biggest cysts was measured with the builtin measuring tool (see Fig. 1b). A posterior vitreous detachment (PVD) was diagnosed by interpreting the vitreoretinal interface. A PVD was present when the vitreous boundary membrane was seen on OCT images.

To see if there are differences regarding the outcome between patients of various visual acuity at the beginning, we divided the cohort into 3 different groups: low: $\leq 60$ (L), middle: 60 up to $\leq 73$ $(\mathrm{M})$, and high: $>73(\mathrm{H})$ letters VA at baseline. Our cohort included quite a few patients with relatively high visual acuity at the beginning of the study in contrast to the cohorts chosen for the prospective studies with an upper limit of 73 letters (20/50). Therefore, we added a third group $(\mathrm{H})$ with $>73$ letters VA at baseline to the usually chosen 2 groups. Within the clinical routine setting, a standard TER without loading dose was used (see online suppl. 1 for full description; for all online suppl. material, see www.karger.com/ doi/10.1159/000514721) and intravitreal injections were applied according to standard procedures [29].

Objectives

The primary objective of the core study was to demonstrate the effectiveness of the TER with respect to the gain in BCVA and the reduction of CRT from baseline to 3-24 months. The primary objective of this publication was within the secondary objective of this study, the analysis of qualitative SD-OCT parameters: their change from baseline to 3-24 months and their impact as outcome predictors with regard to BCVA, BCVA gain, and treatment intervals at 12 and 24 months.

Statistical Analysis

Statistical analysis was performed using SPSS statistical package version 21 (SPSS, Inc., Chicago, IL, USA). For the variables, mean \pm standard deviation or percentages were calculated. To compare follow-up with baseline values, a paired 2 -sided $t$ test was performed. Intergroup comparisons were performed using the $t$ test for independent variables. For correlation analyses, a bivariate Pearson correlation coefficient was evaluated. For all analyses, $p<$ 0.05 was considered statistically significant.

\section{Results}

118 eyes of 87 patients with DME with or without previous laser treatment were reviewed and analyzed retrospectively over a period of 24 months. A subgroup of $28.8 \%(n=34)$ had received a treatment with ranibizumab following a PRN regimen prior to the TER. In this subgroup, the mean duration of treatment following a PRN regimen was $95.76 \pm 73.48$ weeks, and the mean number of intravitreal injections within this regimen was $8.21 \pm$ 6.64. Overall, $43.2 \%$ were not previously treated with laser coagulation. During the follow-up, 74 eyes were newly treated with laser, of which $36.5 \%$ received a focal, $21.6 \%$ a panretinal, and $41.9 \%$ a combined laser treatment.

All eyes were still within ranibizumab TER at 12 months, $82.2 \%$ at 18 months, and $64.4 \%$ at 24 months. And, $9.3 \%$ finished ranibizumab the TER prior to 24 months due to success ( 14 weeks after $3 \times 12$ weeks interval; see online suppl. 1 for TER design), meaning that $73.7 \%$ of eyes were either still the within ranibizumab TER or had finished the ranibizumab TER due to success at 24 months. And, $17.8 \%$ were switched to other substances prior to 24 months due to limited response $(13.6 \%$ aflibercept and 4.2\% dexamethasone implant).

The mean age was $65.93 \pm 11.26$ years. In all, $43.7 \%$ of patients were female and $56.3 \%$ were male. In total, $6.8 \%$ of patients had type 1 diabetes mellitus and the rest (93.2\%) type 2 diabetes mellitus. The mean baseline BCVA of all evaluated eyes was $69.63 \pm 10.84$ ETDRS letters; mean baseline CRT was $404.59 \pm 109.34 \mu \mathrm{m}$. Baseline characteristics are summarized in Table 1.

\section{Best-Corrected Visual Acuity}

There was a significant gain in visual acuity from baseline to 24 months of $4.73 \pm 8.91(p<0.001)$ ETDRS letters (Fig. 2; Table 2 ). The proportion of eyes gaining $\geq 15$ letters was $14.9 \%$ (12 months) and $16.2 \%$ ( 24 months), respectively. At 12 and 24 months, respectively, one patient and two patients lost $\geq 15$ letters, due to a massive increase in intraretinal fluid (IRF), despite a 4-week treatment interval.

Dividing the cohort into 3 different groups (subgroup analysis of low: $\leq 60[\mathrm{~L}]$, middle: 60 up to $\leq 73[\mathrm{M}]$, and 


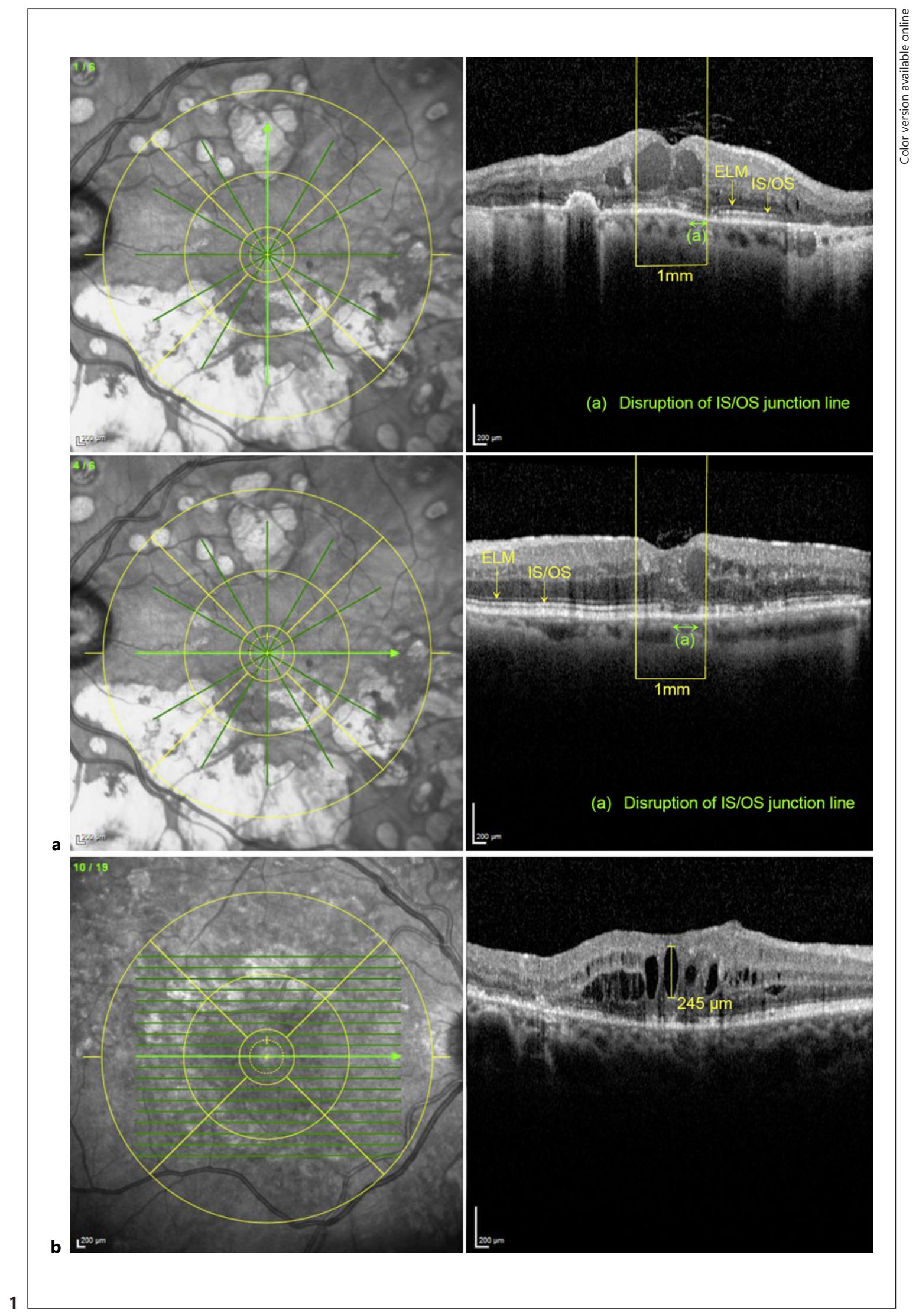

(For legend see next page.) 
Table 1. Patient demographics and disease characteristics at baseline (prior to initiation of TER)

\begin{tabular}{|c|c|}
\hline Characteristic & \\
\hline \multicolumn{2}{|l|}{ Per patient } \\
\hline Mean age $\pm S D$, years & $65.93 \pm 11.26$ \\
\hline \multicolumn{2}{|l|}{ Gender, $n(\%)$} \\
\hline Female & $38(43.7)$ \\
\hline Male & $49(56.3)$ \\
\hline \multicolumn{2}{|l|}{ Diabetes mellitus, $n(\%)$} \\
\hline Type 1 & $8(6.8)$ \\
\hline Type 2 & $79(93.2)$ \\
\hline \multicolumn{2}{|l|}{ Per eye } \\
\hline Mean BCVA \pm SD (letters) & $69.63 \pm 10.84$ \\
\hline Mean CRT $\pm S D, \mu \mathrm{m}$ & $404.59 \pm 109.34$ \\
\hline \multicolumn{2}{|l|}{ DRIL in central $1 \mathrm{~mm}, n(\%)$} \\
\hline Yes & $47(39.8)$ \\
\hline No & $71(60.2)$ \\
\hline \multicolumn{2}{|l|}{ ELM disruption, $n(\%)$} \\
\hline None & $23(19.5)$ \\
\hline Mild & $36(30.5)$ \\
\hline Intermediate & $40(33.9)$ \\
\hline Severe & $19(16.1)$ \\
\hline \multicolumn{2}{|l|}{ IS/OS disruption, $n(\%)$} \\
\hline None & $18(15.3)$ \\
\hline Mild & $50(42.4)$ \\
\hline Intermediate & $34(28.8)$ \\
\hline Severe & $16(13.6)$ \\
\hline Mean number of $\mathrm{HRF} \pm \mathrm{SD}$ & $4.59 \pm 3.26$ \\
\hline \multicolumn{2}{|l|}{ Fluid, $n(\%)$} \\
\hline Intraretinal & $109(92.4)$ \\
\hline Subretinal and intraretinal & $9(7.6)$ \\
\hline \multirow{2}{*}{\multicolumn{2}{|c|}{ Mean height biggest intraretinal cyst \pm SD, $\mu \mathrm{m} \quad 247.42 \pm 129.60$}} \\
\hline & \\
\hline None or incomplete & $81(68.6)$ \\
\hline Complete & $37(31.4)$ \\
\hline \multicolumn{2}{|l|}{ Laser coagulation, $n(\%)$} \\
\hline None & $51(43.2)$ \\
\hline Focal & $16(13.6)$ \\
\hline Panretinal & $12(10.2)$ \\
\hline Focal and panretinal & $39(33.1)$ \\
\hline
\end{tabular}

DRIL, disorganization of inner retinal layer; ELM, external limiting membrane; TER, treat-and-extend regimen; BCVA, best-corrected visual acuity; IS/OS, inner segment/outer segment; CRT, central retinal thickness; HRF, hyperreflective foci; PVD, posterior vitreous detachment; SD, standard deviation.

Fig. 1. a Above a vertical and below a horizontal OCT scan in the macular star setting with the corresponding en face images with an ETDRS grid overlay centered on the fovea. After identification of the central $1 \mathrm{~mm}$ (yellow), the length of the disrupted IS/OS junction line was measured with the measuring tool (green). Disruption was graded as described in the Study Assessments and Treatments section (Methods). Subsequently, the IS/OS disruption score was calculated as the mean of the scores obtained in the hor- high: $>73[\mathrm{H}]$ letters VA at baseline) showed the following increased BCVA at 24 months: $\mathrm{L}$ rose to a mean of $66.32 \pm 8.47, \mathrm{M}$ rose to a mean of $72.60 \pm 10.01$, and $\mathrm{H}$ to a mean of $79.00 \pm 6.35$ letters. The BCVA at 24 months was still significantly different comparing the groups $(\mathrm{L} / \mathrm{M} p=0.045 \mathrm{~L} / \mathrm{H}, p<0.001 \mathrm{M} / \mathrm{H} p=0.017)$. Baseline $\mathrm{VA}$ also had a significant influence on the BCVA gain at 24 months. The group with a middle baseline BCVA had significantly higher BCVA gains at 24 months than the group with a high baseline BCVA (mean $5.80 \pm 9.32$ vs. $0.33 \pm 6.29)(p=0.043)$. Results showed no significant difference between the groups of low and middle baseline BCVA.

Comparing the previous PRN subgroup to treatmentnaive eyes showed that previous treatment based on a PRN regimen before being switched to the TER had no influence on baseline BCVA $(p=0.961)$ and BCVA gain at $12(p=0.602)$ or 24 months $(p=0.726)$. Likewise, a focal or panretinal laser coagulation treatment, neither prior to the TER nor during the follow-up, was not significantly associated with BCVA gain at 12 months $(p=0.069$ and $p=0.416$, respectively) or 24 months ( $p=0.759$ and $p=0.341$, respectively).

\section{IRF and Subretinal Fluid}

Our results show a significant reduction of the CRT from baseline to 24 months of $-99.92 \pm 116.34 \mu \mathrm{m}(p<$ 0.001 ) (Fig. 3; Table 2). There was a constant reduction of CRT from 3 months to 24 months. Eyes with previous PRN treatment prior to the TER did not show significantly different CRT at 24 months $(p=0.144)$ compared to treatment-naive eyes. We found a correlation between baseline CRT and absolute value of CRT decrease at month $12(r=0.595, p<0.001)$ and $24(r=0.772, p<$ $0.001)$, respectively.

There was a significant reduction in the height of the biggest intraretinal cyst from baseline to 24 months, with $-143.75 \pm 159.34(p<0.001)$. At baseline, 9 eyes had subretinal fluid $(7.6 \%)$, improving up to 7 eyes $(5.9 \%)$ at 12 months and $0 \%$ at 24 months. The proportion of eyes be-

izontal and vertical scan, respectively. In this example, the mean disruption score was 1.5 with a score of 1 in the vertical and a score of 2 in the horizontal scan. b Volume OCT scan with an ETDRS grid overlay on the left and measurement of the height of the biggest cyst with the built-in measuring tool on the right. A cyst was defined as a round minimally reflective space within the retina. IS/ OS, inner segment/outer segment; OCT, optical coherence tomography; ELM, external limiting membrane. 
Fig. 2. Graph showing mean change in BCVA from baseline to 24 months with SE. Results show a significant gain in BCVA from baseline throughout the whole observation period $(p<0.001-$ paired sample $t$ test). SE, standard error; BCVA, best-corrected visual acuity.

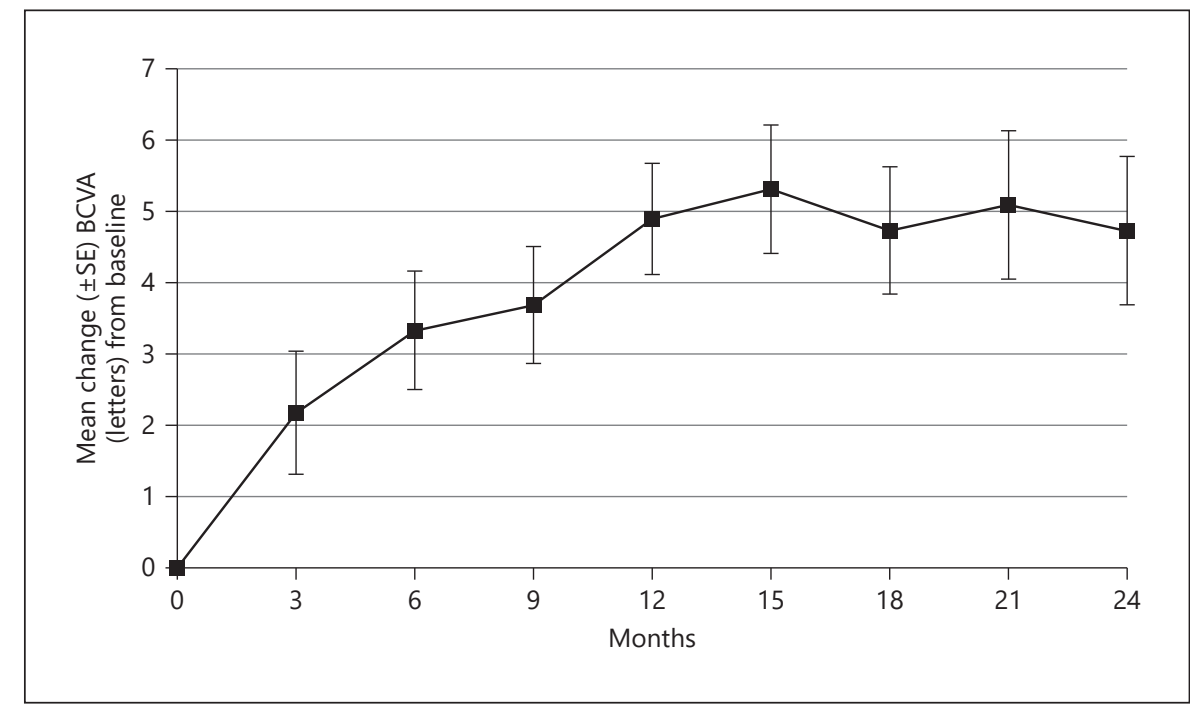

Table 2. Change of clinical and morphologic characteristics during follow-up

\begin{tabular}{lccc}
\hline & Month 12 & Month 18 & Month 24 \\
\hline BCVA gain \pm SD & $4.89 \pm 8.35$ & $4.73 \pm 8.62$ & $4.73 \pm 8.91$ \\
Change of CRT from baseline \pm SD, $\mu \mathrm{m}$ & $-69.63 \pm 109.95$ & $-88.70 \pm 112.36$ & $-99.92 \pm 116.34$ \\
Change of height of biggest cyst \pm SD, $\mu \mathrm{m}$ & $-96.60 \pm 151.65$ & $-123.72 \pm 136.83$ & $-143.75 \pm 159.34$ \\
Proportion of dry OCT, \% & 16.1 & 22.6 & 29.6 \\
Proportion of intact IS/OS interface, \% & 27.1 & 34.0 & 42.1 \\
Proportion of intact ELM, \% & 33.2 & & 34.2 \\
Proportion of DRIL, \% & 31.4 & 2.54 & 21.1 \\
Mean number of HRF & 2.75 & & 51.66 \\
Proportion of PVD, \% & 44.9 & & 51.3 \\
\hline
\end{tabular}

DRIL, disorganization of inner retinal layer; ELM, external limiting membrane; BCVA, best-corrected visual acuity; IS/OS, inner segment/outer segment; OCT, optical coherence tomography; CRT, central retinal thickness; HRF, hyperreflective foci; PVD, posterior vitreous detachment; SD, standard deviation.

ing dry at 12 and 24 months was 16.1 and $29.6 \%$, respectively.

\section{Further OCT Parameters}

At baseline, $15.3 \%$ of eyes showed an intact junction of the photoreceptor's IS/OS junction line, improving up to $27.1 \%$ at 12 months and to $42.1 \%$ at 24 months $(p<0.001)$. Eyes with an impaired IS/OS junction line or ELM (higher disruption score) at baseline had a worse BCVA at 12 months $(r=-0.347, p<0.001$, and $r=-0.476, p<0.001$, respectively). We also found a negative correlation between the IS/OS and ELM disruption score at 12 months and BCVA at 24 months $(r=-0.255, p=0.027$ and $r=$ $-0.528, p<0.001$, respectively), meaning that eyes with higher disruption score (more disruption) at 12 months showed worse BCVA at 24 months. Eyes exhibiting a DRIL in the central $1 \mathrm{~mm}$ at baseline had a significantly worse BCVA at 12 and 24 months $(p=0.003$ and $p=$ 0.047 , respectively). Patients with a higher biggest cyst at baseline showed a worse disruption score of the IS/OS junction line at baseline $(r=0.538, p<0.001)$ as well as at 12 months $(r=0.299, p=0.001)$ and 24 months $(r=0.305$, $p=0.007)$. Likewise, a higher CRT at baseline was correlated with a more impaired IS/OS junction line at baseline $(r=0.526, p<0.001)$ and at 12 months $(r=0.280, p=$ $0.002)$.

The mean number of HRFs as the sum of counts in the vertical and horizontal scans within the central $1 \mathrm{~mm}$ was reduced from 4.59 at baseline to 1.66 at 24 months $(p<$ 0.001 ), with $92.4 \%$ of eyes showing HRFs at baseline with- 
Fig. 3. Graph showing mean change in CRT in percent from baseline to 24 months with SD. Results indicate a significant reduction in CRT throughout the whole observation period $(p<0.001-$ paired sample $t$ test). SD, standard deviation; CRT, central retinal thickness; SE, standard error.

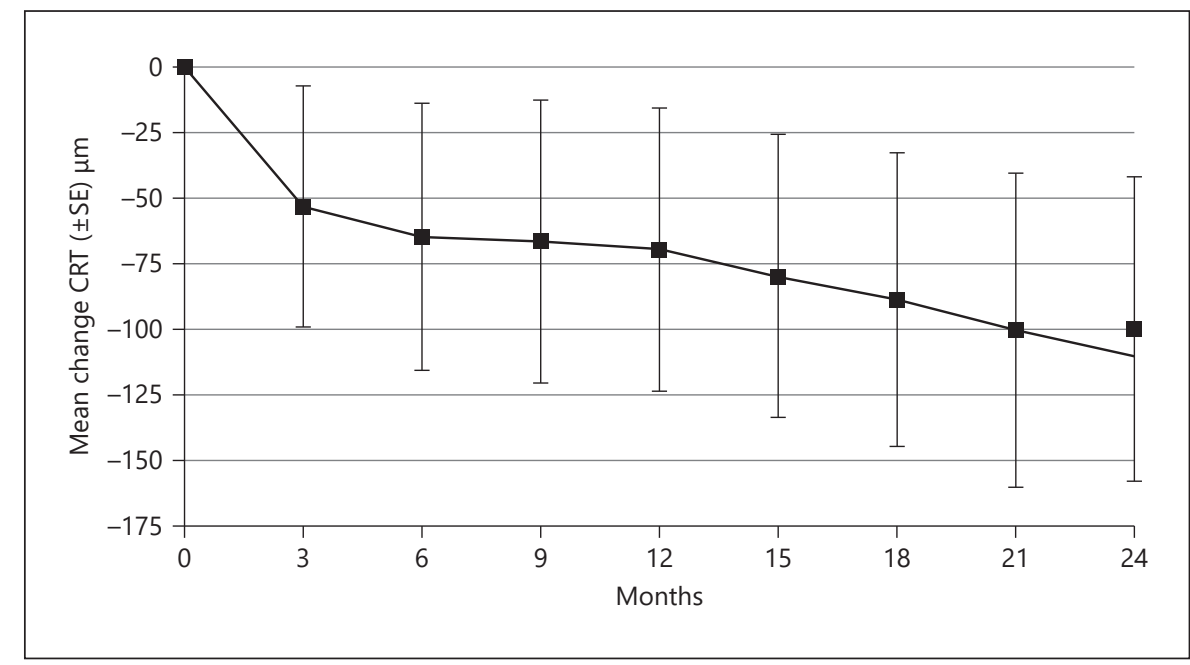

in these scans in the central $1 \mathrm{~mm}$ and $84.2 \%$ at 24 months. As evaluated by SD-OCT at baseline, $31.4 \%$ of the eyes had a complete PVD, increasing up to $51.3 \%$ at 24 months. Comparison concerning the number of injections in eyes with or without baseline PVD did not show a significant difference $(10.19 \pm 2.1$ with total PVD vs. $9.47 \pm 2.17$ with partial or no PVD, $p=0.094$ ).

\section{Number of Injections and Treatment Intervals}

Patients in the second year of treatment received significantly less injections than during the first 12 months $(7.88 \pm 2.59$ vs. $9.69 \pm 2.17$ injections, $p<0.001)$. The mean of the individual maximal RFTIs was $6.73 \pm 2.81$ weeks during the first 12 months and $7.57 \pm 3.18$ weeks between 12 and 24 months $(p<0.001)$. Maximal RFTI during the whole observation period of 24 months was $7.13 \pm 2.75$ weeks. At 24 months, $25.0 \%$ of patients $(n=$ $25)$ reached the maximum RFTI of 12 weeks. (For distribution of treatment intervals at 12 and 24 months, see Figure 4.)

If the junction of the photoreceptor's IS/OS junction was intact at baseline (low IS/OS disruption score), longer maximum RFTIs were reached $(r=-0.345, p<0.001)$. If the baseline IRF was $>350 \mu \mathrm{m}$ (maximum height of biggest cyst), RFTIs were shorter at 12 months $(r=-0.425$, $p<0.001)$.

\section{Logistic Regression Analysis}

We analyzed 4 baseline parameters (number of HRFs, integrity of IS/OS junction line, size of biggest cyst, and age) to predict the probability of having a dry retina after 12,18 , and 24 months. Results indicate that only the in-

Predictors for Outcome of TER in DME

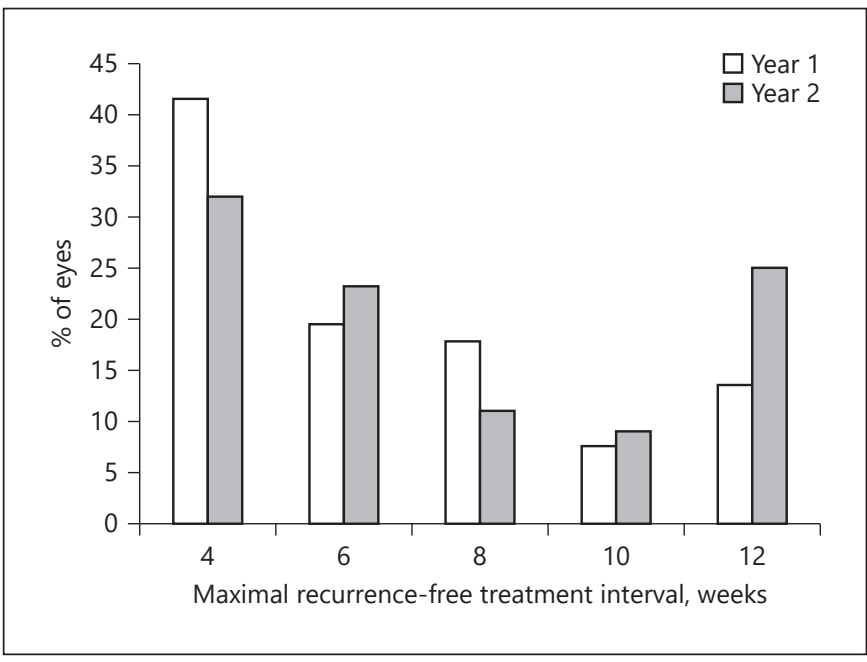

Fig. 4. Column graph comparing the distribution of maximal RFTI, at 12 (year 1) and 24 (year 2) months. Patients had significantly longer maximal RFTIs during the second year $(p<0.001-$ paired sample $t$ test). RFTI, recurrence-free treatment interval.

tegrity of the IS/OS junction line plays a role as a predicting factor. The other 3 parameters did not show significance. Specifically, that means that patients having an intact IS/OS junction line at baseline increased their probability of having a dry retina after 12 months by $79.3 \%(p=0.017)$, after 18 months by $82.0 \%(p=0.040)$, and after 24 months by $88.1 \%(p=0.040)$ with the treatment mentioned previously.

A multiple regression analysis was run to predict BCVA and BCVA gain throughout the study period from 
baseline BCVA, integrity of IS/OS junction line, size of the biggest cyst, the number of HRFs, and age. The prediction for BCVA gain at 24 months showed that only baseline BCVA and age added significantly to the prediction $(p<0.001$ and $p=0.046$, respectively). Specifically, the BCVA gain at 24 months decreased by 0.401 with every increase of 1 letter of baseline BCVA and decreased by 0.158 with every year a patient was older. The analysis for BCVA at 24 months also showed that only baseline BCVA and age were significant predictors $(p<0.001$ and $p=0.046$, respectively). Hereby, an increase of 1 letter of baseline BCVA was associated with an increase by 0.599 of BCVA at 24 months. With every year a patient was older, BCVA at 24 months decreased by 0.158 .

\section{Discussion}

Results of this study show that this TER without a loading dose resulted in a significant improvement of visual acuity and anatomic features in patients with DME over 24 months, with increasing treatment intervals from year 1 to 2. In nAMD and macular edema due to RVO, the TER showed to be associated with higher injection rates than PRN $[16,18]$. The TER in the RETAIN [20] study led to a lower number of injections during both the first and second years of treatment than this study (7 vs. 9.7 and 5 vs. 7.9 injections). The main reason for that is certainly that in the RETAIN study, extension criteria had been more tolerant and intervals had been extended in larger steps [20]. For the total 24-month follow-up period, our injection frequency is almost exactly the same like in the TREX-DME trial [21]. The 12-month analysis of a mixed ranibizumab/aflibercept clinical routine TER setting by Schwarzer et al. [25] showed comparable injection numbers of about 10 for the first year. The mean maximum treatment interval in their setting was 8.5 weeks, while we reported a mean maximum RFTI of 6.7 weeks, which corresponds to a mean maximum "tried" treatment interval of 8.7 weeks using extensions in 2-week steps. Both clinical routine settings did not use a loading dose, allowing for earlier extension in case of rapid responding smaller edema. However, the high rate of patients with 4 -week treatment intervals and a mean injection rate of about 10 during year 1 reflect the need for high-frequency treatment in many DME eyes compared to an average exudative AMD population. The significantly increasing treatment intervals throughout year 2 show the characteristic behavior of consequently treated DME. However, despite following a strict TER without loading phase but with immediate interval extension in case of DME inactivity and exit strategy, an overtreatment in several cases cannot be ruled out completely.

An analysis across 9 phase II and III randomized clinical trials of ranibizumab $0.3 / 0.5 \mathrm{mg}$ and aflibercept $2 \mathrm{mg}$ has demonstrated that regardless of the regimen and the anti-VEGF compound, the mean visual acuity at 12 months plateaued at about 70 letters [30]. In our study, visual acuity plateaued almost at 75 letters after 12 months, which is about 1 line above most prospective trials [30]. This might have been influenced by the much better baseline visual acuity in our population than that in most prospective trials [30]. Our VA gain was with about 5 letters lower after 12 and 24 months than in all the major studies (up to 12 letters there). The reasons are our much higher BL BCVA (70 letters) than these studies (mostly around 60 , up to 64 letters) and our "routine clinical setting" without exclusion of small edema. Probably due to the same reasons in our study, the proportion of eyes gaining $\geq 15$ letters was only $14.9 \%$ (12 months) and $16.2 \%$ (24 months), respectively. In the RISE and RIDE, patients treated with ranibizumab achieved earlier vision gains with over $50 \%$ gaining $\geq 15$ ETDRS letters within 12 months [20,31]. Like in the RETAIN study [20], we could show that greater BCVA gains can be observed in patients with poorer visual acuity at baseline. This is also called the ceiling effect: patients with a poor VA at baseline have a higher scope to achieve clinically meaningful visual gains than those starting off with already high VA scores over the course of treatment [31]. In the RESTORE study, no differences were detected between the ranibizumab and the ranibizumab plus laser arms regarding BCVA outcomes [12]. This is in accordance with our study with focal or panretinal laser coagulation treatment, neither prior to the TER nor during the follow-up, being not significantly associated with BCVA gain at 12 or 24 months.

Reviewing the literature, it is generally stated that age and sex influence final VA in DME patients with antiVEGF therapy [32]. In our study, higher age predicted both less visual acuity gain and worse visual acuity at 24 months. After adjusting for baseline VA in a post hoc analysis of the Protocol I Study [33], the study group found that younger age, less severe diabetic retinopathy, and the absence of surface wrinkling retinopathy were associated with a greater benefit in final VA. Our results reflect the ones found in the literature, such that a relationship exists between baseline VA, age, and final VA in DME patients treated with ranibizumab.

Eski Yucel et al. [34] reported in their study that age, gender, baseline VA, and 3 OCT findings are associated 
with a 2-year visual outcome: vitreomacular traction, PVD, and disruption of the ellipsoid zone. In our study, the disruption of the IS/OS line and ELM within the central $1 \mathrm{~mm}$ showed a negative correlation with BCVA at 12 months. We also found a negative correlation between the IS/OS junction line and ELM disruption scores at 12 months and BCVA at 24 months. The latter indicates that eyes which show impaired IS/OS and ELM zones after having received the first treatments have less favorable visual acuity outcomes over the long-term. As previously shown, evaluation of IS/OS and ELM integrity is often difficult at baseline due to the more severe edema with a lot of cysts and HRFs, while quantification seems to be easier and therefore better correlated with long-term BCVA, using OCT scans after the induction phase [28]. Further, patients having an intact IS/OS junction line at baseline had a higher chance of having a dry retina after 2 years of treatment, which might be caused by less severe edema in patients with intact IS/OS line at baseline.

Cystoid changes beneath the outer plexiform layer have been shown to present risk to photoreceptor cells and to impair IS/OS integrity [35]. This fact was also demonstrated in our study with a correlation of biggest cyst height at baseline with the disruption score of IS/OS junction line at baseline. Further, biggest cyst height was a predictor for IS/OS disruption at 12 and 24 months. In 2012, Murakami et al. [35] investigated the relationship of cystoid spaces in the outer retinal layers (especially the OPL) and the characteristics of the photoreceptors beneath these cystoid spaces in patients with DME. They found that the areas beneath cystoid spaces in the OPL had a longer length of disrupted IS/OS and disrupted external membrane (ELM) line than those without cystoid spaces, suggesting that cystoid spaces in the OPL might be an important mechanism for photoreceptor damage. Gerendas et al. [36] reported from a RESTORE post hoc analysis that the height of intraretinal cystoid spaces at baseline is a better predictor of functional/anatomical improvement than CRT alone. In 2016, another study group [37] assessed the relationship between SD-OCT features and functional outcomes for DME under bevacizumab PRN and found that both the more severe IRF and the better ellipsoid zone integrity predicted more visual acuity gain.

The amount of HRFs or hard exudates changes after treatment for DME [38]. In 2012, a study [38] noted a significantly reduced number of HRFs, a reduced foveal thickness, and an increased visual acuity in patients with DME treated with bevacizumab or ranibizumab. But no distinct overall correlation was found between the HRF reduction and the course of visual acuity or retinal thickness. These results are in keeping with our findings. We could show a significant reduction in the mean number of HRF after 1 year of treatment, without them having a significant influence on visual acuity or retinal thickness after 2 years of treatment following our TER protocol. Bressler et al. [33] stated that a year after treatment of DME with ranibizumab, although hard exudates were associated with a more favorable improvement on OCT scans, they were not associated with vision outcome. Other studies, like the DRCR Retina Network Protocol-I Study, found that hard exudates were correlated with both higher VA gain and anatomical improvement [19].

Finally, our study evaluated DRIL as a newly described SD-OCT feature in DME [39]. In our study, eyes exhibiting a DRIL in the central $1 \mathrm{~mm}$ at baseline had a significantly worse BCVA at 12 and 24 months. Sun et al. [39] described in a cross-sectional study a strong correlation between DRIL and visual acuity in eyes with present or resolved DME. For steroid treatment, DRIL seems to have a high predictive value in regard to visual outcomes [40]. Unfortunately, so far, there are limited data regarding DRIL as an outcome predictor in consequent longterm anti-VEGF treatment for DME. It should be considered as a SD-OCT parameter for upcoming trials.

Strengths of this study are the highly standardized routine care procedures within our TER setting including imaging, examination, documentation, and applying TER. Further, all patients received the same drug, and for a routine clinical setting, the treatment adherence was high up to 24 months. Limitations of the study are its retrospective design, the lack of a control group and several dropouts mainly due to switching to other treatments within this clinical routine setting. OCT features were analyzed by 2 independent physicians. Even if readers received a standardized training and a standardized protocol was followed, intergrader differences cannot be ruled out. Further, finer OCT segmentation might have shown more details that our clinical routine setting segmentation could have missed.

To conclude, ranibizumab applied in a TER without loading dose in DME significantly improves visual acuity and retinal anatomical structure throughout 2 years. An intact IS/OS junction at baseline seems to be a predictor for a dry retina later under treatment. Less IS/OS junction disruption at baseline predicted longer maximum RFTIs at 2 years and better visual acuity at 1 year. More severe edema with bigger intraretinal cysts and thicker retina was associated with more IS/OS disruption. DRIL in the central $1 \mathrm{~mm}$ at baseline predicted worse visual acuity 
outcomes. Other OCT features like HRF and PVD did not have predicting powers in this study. Younger patients and a lower VA at baseline were predictive of a higher final VA gain. These predictors might help guide routine clinical treatment in DME.

\section{Acknowledgements}

The authors thank Mrs. Susanne Müller for her valuable support during document preparation for ethics approval, data collection, and manuscript submission.

\section{Statement of Ethics}

Ethical approval of the study was obtained from the Local Ethics Committee (Ethikkommission Nordwestschweiz [EKNZ No 2014-322]). The study was conducted in accordance with all applicable laws and regulations, and ICH-GCP guidelines were followed whenever applicable.

\section{Conflict of Interest Statement}

C.P. worked as consultant for and received financial support from Alcon, Bayer, Novartis, and Oertli. K.H. received financial support from Bayer, Novartis, Allergan, and Roche and worked as consultant for Kinarus AG. All the other authors have no conflicts of interest/competing interests to declare.

\section{Funding Sources}

This is an industry-independent study performed as a doctoral thesis (Alexandra Behrndt) with the resources and support from Vista Klinik Binningen, Switzerland. There was no external funding.

\section{Author Contributions}

H.G.Z.: interpretation of data and writing of the manuscript. A.B.: acquisition, interpretation of data, statistics, and writing of the manuscript. L.H.: acquisition, interpretation of data, and statistics. M.M.G.: interpretation of data and writing of the manuscript. C.T.: image processing supervision and statistics. C.P.: design of the study and interpretation of data. K.H.: design of the study, acquisition, interpretation of data, supervision, and writing of the manuscript. All authors critically reviewed the manuscript and approved the final version.

\section{Availability of Data and Material}

Due to the small sample size, patient-level data will not be made available in order to ensure that patient privacy is safeguarded.

\section{Consent to Participate/for Publication}

The need for written consent from each individual patient was waived because of the retrospective nature of the study.

\section{References}

1 Klein R, Klein BE, Moss SE. Visual impairment in diabetes. Ophthalmology. 1984;91(1):1.

2 Antonetti DA, Klein R, Gardner TW. Diabetic retinopathy. N Engl J Med. 2012;366(13): 1227.

3 Klein R, Klein BE, Moss SE, Davis MD, DeMets DL. The Wisconsin epidemiologic study of diabetic retinopathy: III. Prevalence and risk of diabetic retinopathy when age at diagnosis is 30 or more years. Arch Ophthalmol. 1984;102(4):527-32.

4 Schmidt-Erfurth U, Garcia-Arumi J, Bandello F, Berg K, Chakravarthy U, Gerendas BS, et al. Guidelines for the management of diabetic macular edema by the European Society of Retina Specialists (EURETINA). Ophthalmologica. 2017;237(4):185.

5 Silva PS, Sun JK, Aiello LP. Role of steroids in the management of diabetic macular edema and proliferative diabetic retinopathy. Semin Ophthalmol. 2009;24(2):93-9.

6 Çekiç O, Chang S, Tseng JJ, Akar Y, Barile GR, Schiff WM. Cataract progression after intravitreal triamcinolone injection. Am J Ophthalmol. 2005;139(6):993-8.
7 Wingate RJ, Beaumont PE. Intravitreal triamcinolone and elevated intraocular pressure. Aust N Z J Ophthalmol. 1999;27(6):431.

8 Boyer DS, Yoon YH, Belfort R, Bandello F, Maturi RK, Augustin AJ, et al. Three-year, randomized, sham-controlled trial of dexamethasone intravitreal implant in patients with diabetic macular edema. Ophthalmology. 2014;121(10): 1904.

9 Pearson PA, Comstock TL, Ip M, Callanan D, Morse LS, Ashton P, et al. Fluocinolone acetonide intravitreal implant for diabetic macular edema: a 3-year multicenter, randomized, controlled clinical trial. Ophthalmology. 2011;118(8):1580.

10 Kim EJ, Lin WV, Rodriguez SM, Chen A, Loya A, Weng CY. Treatment of diabetic macular edema. Curr Diab Rep. 2019;19(9): 68.

11 Nguyen QD, Brown DM, Marcus DM, Boyer DS, Patel S, Feiner L, et al. Ranibizumab for diabetic macular edema: results from 2 phase iii randomized trials: RISE and RIDE. Ophthalmology. 2012;119(4):789.
12 Mitchell P, Bandello F, Schmidt-Erfurth U, Lang GE, Massin P, Schlingemann RO, et al. The RESTORE study: ranibizumab monotherapy or combined with laser versus laser monotherapy for diabetic macular edema. Ophthalmology. 2011;118(4):615.

13 Elman MJ, Elman MJ, Qin H, Aiello LP, Beck $\mathrm{RW}$, Bressler NM, et al. Intravitreal ranibizumab for diabetic macular edema with prompt versus deferred laser treatment: three-year randomized trial results. Ophthalmology. 2012;119(11):2312.

14 Spaide R. Ranibizumab according to need: a treatment for age-related macular degeneration. Am J Ophthalmol. 2007;143(4):679-80.

15 Hatz K, Prünte C. Changing from a pro re nata treatment regimen to a treat and extend regimen with ranibizumab in neovascular age-related macular degeneration. $\mathrm{Br} \mathrm{J}$ Ophthalmol. 2016;100(10): 1341.

16 Hatz K, Prünte C. Treat and extend versus pro re nata regimens of ranibizumab in neovascular age-related macular degeneration: a comparative 12 month study. Acta Ophthalmol. 2017;95(1):e67. 
17 Freund KB, Korobelnik JF, Devenyi R, Framme C, Galic J, Herbert E, et al. Treatand-extend regimens with anti-VEGF agents in retinal diseases: a literature review and consensus recommendations. Retina. 2015;35(8): 1489.

18 Guichard MM, Xavier AR, Türksever C, Pruente C, Hatz K. Spectral-domain optical coherence tomography-driven treat-and-extend and pro re nata regimen in patients with macular oedema due to retinal vein occlusion: 24-month evaluation and outcome predictors. Ophthalmic Res. 2018;60(1):29-37.

19 Baker CW, Jiang Y, Stone T. Recent advancements in diabetic retinopathy treatment from the diabetic retinopathy clinical research network. Curr Opin Ophthalmol. 2016;27(3): 210.

20 Prünte C, Fajnkuchen F, Mahmood S, Ricci F, Hatz K, Studnička J, et al. Ranibizumab 0.5 $\mathrm{mg}$ treat-and-extend regimen for diabetic macular oedema: the RETAIN study. Br J Ophthalmol. 2016;100(6):787.

21 Payne JF, Wykoff CC, Clark WL, Bruce BB, Boyer DS, Brown DM, et al. Randomized trial of treat and extend ranibizumab with and without navigated laser versus monthly dosing for diabetic macular edema: TREX-DME 2-year outcomes. Am J Ophthalmol. 2019; 202:91-9.

22 Volkmann I, Knoll K, Wiezorrek M, Greb O, Framme C. Individualized treat-and-extend regime for optimization of real-world vision outcome and improved patients' persistence. BMC Ophthalmol. 2020;20(1):122.

23 Curry BA, Sanfilippo PG, Chan S, Hewitt AW, Verma N. Clinical outcomes of a treat and extend regimen with intravitreal aflibercept injections in patients with diabetic macular edema: experience in clinical practice. Ophthalmol Ther. 2020;9(1):87-101.

24 Sugimoto M, Ichio A, Nunome T, Kondo M. Two year result of intravitreal bevacizumab for diabetic macular edema using treat and extend protocol. Medicine. 2017;96(16): e6406.
25 Schwarzer P, Ebneter A, Munk M, Wolf S, Zinkernagel MS. One-year results of using a treat-and-extend regimen without a loading phase with anti-VEGF agents in patients with treatment-naive diabetic macular edema. Ophthalmologica. 2019;241(4):220-5.

26 Rayess N, Rahimy E, Ying GS, Bagheri N, Ho $\mathrm{AC}$, Regillo $\mathrm{CD}$, et al. Baseline choroidal thickness as a predictor for response to antivascular endothelial growth factor therapy in diabetic macular edema. Am J Ophthalmol. 2015;159(1):85.

27 Gregori NZ, Feuer W, Rosenfeld PJ. Novel method for analyzing snellen visual acuity measurements. Retina. 2010;30(7):1046-50.

28 Hatz K, Ebneter A, Tuerksever C, Pruente C, Zinkernagel M. Repeated dexamethasone intravitreal implant for the treatment of diabetic macular oedema unresponsive to antiVEGF therapy: outcome and predictive SDOCT features. Ophthalmologica. 2018; 239(4):205-14.

29 Grzybowski A, Told R, Sacu S, Bandello F, Moisseiev E, Loewenstein A, et al. 2018 update on intravitreal injections: euretina expert consensus recommendations. Ophthalmologica. 2018;239(4):181-93.

30 Dugel PU, Hillenkamp J, Sivaprasad S, Vögeler J, Mousseau MC, Wenzel A, et al. Baseline visual acuity strongly predicts visual acuity gain in patients with diabetic macular edema following anti-vascular endothelial growth factor treatment across trials. Clin Ophthalmol. 2016;10:1103.

31 Morse L, Yau L, Tuomi L. Clinically meaningful visual improvements and predictors of early vision gains with ranibizumab for diabetic macular oedema. BMJ Open Ophthalmol. 2019;4(1):e000335.

32 Ashraf M, Souka A, Adelman R. Predicting outcomes to anti-vascular endothelial growth factor (VEGF) therapy in diabetic macular oedema: a review of the literature. Br J Ophthalmol. 2016;100(12):1596.
33 Bressler SB, Qin H, Beck RW, Chalam KV, Kim JE, Melia M, et al. Factors associated with changes in visual acuity and central subfield thickness at 1 year after treatment for diabetic macular edema with ranibizumab. Arch Ophthalmol. 2012;130(9):1153.

34 Eski Yucel O, Birinci H, Sullu Y. Outcome and predictors for 2-year visual acuity in eyes with diabetic macular edema treated with ranibizumab. J Ocul Pharmacol Ther. 2019;35(4): 229-34.

35 Murakami T, Nishijima K, Akagi T, Uji A, Horii T, Ueda-Arakawa N, et al. Optical coherence tomographic reflectivity of photoreceptors beneath cystoid spaces in diabetic macular edema. Invest Ophthalmol Vis Sci. 2012;53(3): 1506.

36 Gerendas BS, Prager S, Deak G, Simader C, Lammer J, Waldstein SM, et al. Predictive imaging biomarkers relevant for functional and anatomical outcomes during ranibizumab therapy of diabetic macular oedema. Br J Ophthalmol. 2018;102(2):195-203.

37 Itoh Y, Petkovsek D, Kaiser PK, Singh RP, Ehlers JP. Optical coherence tomography features in diabetic macular edema and the impact on anti-VEGF response. Ophthalmic Surg Lasers Imaging Retin. 2016;47(10):90813.

38 Framme C, Schweizer P, Imesch M, Wolf S, Wolf-Schnurrbusch U. Behavior of SD-OCTdetected hyperreflective foci in the retina of anti-VEGF-treated patients with diabetic macular edema. Invest Ophthalmol Vis Sci. 2012;53(9):5814.

39 Sun JK, Radwan SH, Soliman AZ, Lammer J, Lin MM, Prager SG, et al. Neural retinal disorganization as a robust marker of visual acuity in current and resolved diabetic macular edema. Diabetes. 2015 Jul;64(7):2560-70.

40 Zur D, Iglicki M, Sala-Puigdollers A, Chhablani J, Lupidi M, Fraser-Bell S, et al. Disorganization of retinal inner layers as a biomarker in patients with diabetic macular oedema treated with dexamethasone implant. Acta Ophthalmol. 2020 Mar;98(2):e217-23. 